\title{
TRAVELLING WAVES IN A MEDIUM WITH NON-LINEAR HEAT CONDUCTION*
}

\author{
A. A. SAMARSKII, S.P. KURDYUMOV and P.P. VOLOSEVICH** \\ Moscow \\ (Received 8 June 1964)
}

\section{Introduction}

THF equations of non-linear heat conduction have been investigated in [1] - [5]. It was established that the dependence of the thermal conductivity on the temperature led to the possibility of heat propagation (with zero initial background temperature) at a finite speed. It is interesting to clarify how the peculiarities of non-linear heat conduction inanifest themselves as the interaction of thermal and hydrodynamic processes.

It is well known that the processes described by the hydrodynamic equations for non-linear heat conduction are sharacteristic for problems of radiational hydrodynamics (see [4]) where the interaction of radiation with matter is considered. On the assumption of a local thermal equilibrium of radiation with matter, and also by neglecting radiation pressure in comparison with material pressure, the presence of radiation in the medium appears only in abnormally great heat conduction of the medium and in the dependence of the thermal conductivity on the temperature. The molecular process of transfer in such a medium (i.e. ordinary viscosity and heat conduction) can be neglected in comparison with the heat conduction caused by radiation. High thermal conductivity of the medium in a series of cases, as has been shown in [4] and [5], leads to the appearance of temperature wave (TW). TW are divided into TW-1 (heat

- Zh. vȳchisl, Mat, mat, Fiz, 5, 2, $199-217,1965$.

* The basic theoretical content of this paper was presented by the authors at the Fourth All-Union Mathenatical Congress in 1961. 
propagation with supersonic speed) and TW-2 (subsonic heat propagation) according to the type of hydrodynamic motion which they produce. See $[4,6,7]$.

The given approach to problems of temperature waves was to some extent new because generally, in considering hydrodynamic problems with heat conduction, purely hydrodynamic phenomena were introduced into the first plan and heat conduction only played the role of a dissipating and smoothing out factor.

Thus in [8] the consideration of the processes of ordinary molecular viscosity and heat conduction was used to study the structure of a shock wave (SW) front. As is well known, if the dissipative processes are not taken into account in a medium, the SW becomes a strong discontinuity. Consideration of heat conduction leads, as was shown in [8], to the formation of a definite effective "width" to the wave.

In this paper a self-modelling of a type of travelling wave is used, but as distinct from [8] the case of non-linear heat conduction is studied and the problem of a travelling wave is connected with the problem of a piston with a thermal system. On account of this approach we have been successful in investigating not only the structure of a travelling wave, accomplishing the passage from one constant solution to another, but also the more general form of a travelling wave, essentially connected with a non-stationary thermal and hydrodynamic system for a piston.

Within the scope of a one-dimensional plane problem for hydrodynamic equations with non-linear heat conduction (the thermal conductivity $x=x_{0} T^{m} \rho^{\alpha}$, where $T$ is the temperature and $\rho$ the density) we consider the problem of a piston with a defined law of variation of heat flow and speed of the piston with time, bringing about the formation of a travelling wave in fronl of the piston. At the initial instant it is assumed that there is a null temperature, speed and pressure background and a value of the density which is constant in the space.

In this paper some types of continuous and discontinuous travelling waves are constructed.

The majority of the waves formed exist only up to a definite instant of time. Together with metastable waves a traveling wave is formed which unites two constant solutions and has a finite width for the transition zone. In this paper the relation between this width and the parameters of the problem is established. The structure of a SW in a medium with radiation, bringing in the kinetic equations for the radiation, was 
investigated in more detail in [9]. We consider the problem stated above about a travelling wave as a model example, which enables us to analyse the possible types of solution and their dependence on the degree of non-linearity of the equation of heat conduction. In this connection, in the problem the analysis is given of a field of integral curves depending on the values of the exponents of non-linearity $m$ and $\sigma$ and also on the value of $\gamma=c_{p} / c_{V}$. In a number of cases we can form an analytic solution. The given analysis is used to construct and classify various types of travelling temperature waves.

Together with the construction of self-modelling solutions we quote here the results of calculations of number of solutions obtained on a computer by means of the difference method of solving a system of partial differential equations with corresponding boundary conditions on the piston.

The composition of the self-modelling and numerical solutions enables us, on the one hand, to form an opinion of the accuracy of the difference methods used and, on the other, it confirms the existence and stability of the series of travelling waves constructed.

\section{Formulation of the problem}

The problem is considered in the plane one-dimensional case with the equation of state

$$
p=R \rho T, \quad \varepsilon=c_{V} T=\frac{R}{\gamma-1} T .
$$

The system of gas dynamic equations with non-linear heat conduction is of the form

$$
\begin{gathered}
\frac{\partial v}{\partial t}=-\frac{\partial p}{\partial x}, \quad \frac{\partial \eta}{\partial t}=\frac{\partial v}{\partial x} \\
\frac{\partial \varepsilon}{\partial t}=-p \frac{\partial v}{\partial x}-\frac{\partial w}{\partial x}, \quad w=-\varkappa_{0} T^{m} \eta^{\sigma} \frac{\partial T}{\partial x}
\end{gathered}
$$

where $x$ is the mass coordinate expressed in grames, $\eta=1 / \rho$ the specific volume, $p$ the pressure, $v$ the speed, $T$ the temperature, $w$ the heat flux and $\kappa, F$ and $\gamma$ are constants. The solution is sought in the form of a travelling wave propagated with constant mass speed $D \mathrm{~g} / \mathrm{sec}$. It is assumed that on the background in front of the wave we are given the 
following initial values:

$$
\rho=\rho_{0}, \quad v=v_{0}, \quad T=0, \quad w=0
$$

The system of equations which describes the self-modelling solution of the type of travelling wave is obtained from (1), changing to a new variable. It is convenient to write the system considered in dimensionless form. For this we express the scales of magnitude by the constant defining parameters of the problem, which have independent dimensionality, e.g. by $R, \kappa_{0}, D$ and $\rho_{0}$. The dimensionless self-modelling variable will have the form

$$
\xi=\frac{D t-x}{\varkappa_{0} D^{2 m-1} R^{-(m+1)} \rho_{0}^{-(2 m+\sigma)}} .
$$

The transition to dimensionless functions is given by the following formulae

$$
\begin{gathered}
\bar{v}(\xi)=\frac{v(x, t)}{D \rho_{0}^{-1}}, \quad \bar{p}(\xi)=\frac{p(x, t)}{D^{2} \rho_{0}^{-1}}, \quad \bar{w}(\xi)=\frac{w(x, t)}{D^{3} \rho_{0}^{-2}}, \\
\bar{T}(\xi)=\frac{T(x, t)}{D^{2} R^{-1} \rho_{0}^{-2}}, \quad \bar{\eta}(\xi)=\frac{\rho_{0}}{\rho(x, t)}=\rho_{0} \eta(x, t) .
\end{gathered}
$$

Using (3) and (4) we obtain from (1) a system of ordinary differential equations

$$
\begin{gathered}
\frac{d \bar{v}}{d \xi}=\frac{d \bar{p}}{d \xi}, \quad \frac{d \bar{\eta}}{d \xi}=-\frac{\overline{d v}}{d \xi}, \quad \bar{p}=\frac{\bar{T}}{\bar{\eta}}, \\
\frac{d\left(\frac{1}{\gamma-1} \bar{T}\right)}{d \xi}=\bar{p} \frac{d \bar{v}}{d \xi}+\frac{d \bar{w}}{d \xi}, \quad \bar{w}=\bar{T}^{m} \overline{\eta^{\sigma}} \frac{d \bar{T}}{d \xi} .
\end{gathered}
$$

The system ( $\left.1^{\prime}\right)$ permits of three integrals

$$
\bar{v}=\bar{v}_{0}+1-\eta, \quad \ddot{p}=1-\bar{\eta}_{;} \quad \bar{w}=0.5\left(\frac{\gamma+1}{\gamma-1} \bar{\eta}-1\right)(1-\bar{\eta}),
$$

and the expression

$$
\bar{T}=\bar{\eta}(1-\bar{\eta})
$$

is valid.

To obtain (5) the integration is carried out from the backgraund in front of the wave on which the given conditions are $\bar{\eta}=\bar{\eta}_{0}=1, \quad \bar{v}=\bar{v}_{0}, \quad \bar{T}=\bar{T}_{0}=0, \quad \bar{p}=\bar{p}_{0}=0, \quad \bar{w}=\bar{w}_{0}=0$. 
As a result system (1') reduces to the algebraic relations (5) and one differential equation

$$
\bar{\eta}^{\prime}=0 . \bar{\zeta} \frac{[(\gamma+1 /(\gamma-1)] \bar{\eta}-1}{(1-\bar{\eta})^{m-1} \bar{\eta}^{0+m}(1-2 \bar{\eta})}=F(\bar{\eta}) .
$$

It will be shown below that the initial value $\bar{\eta}=\eta_{0}=1$ is given efther at a finite point $\xi=\xi_{0}$ or with $\xi_{0}=-\infty$, depending on the region of variation of the paraneter $m$.

In the first case the wave has a front which spreads with a finite speed relative to the initial background. The invariance of equation (6)

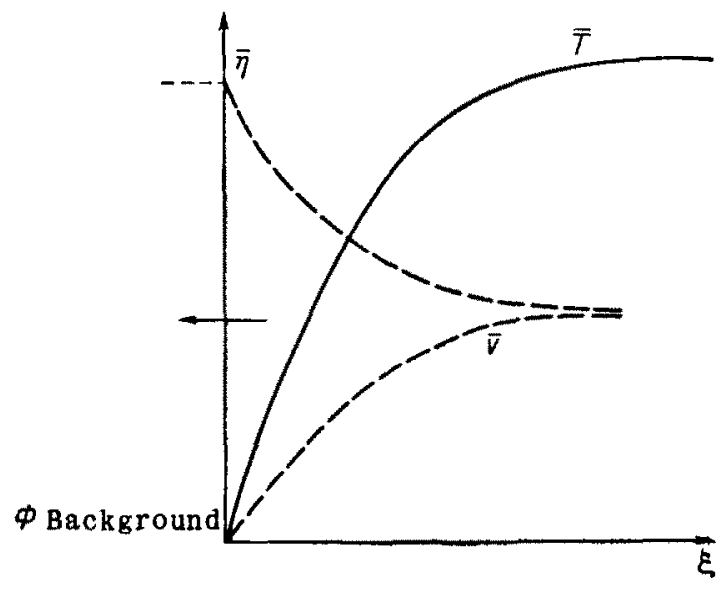

FIG. 1 .

with respect to the transformation $\xi^{\prime}=\xi+\xi_{0}$ enables us to locate the wave front at the point $\xi=\xi_{0}=0$. Since $\xi \sim(D t-x)$ the wave front is transferred in accordance with the law $x_{\xi_{0}}=D t$.

We can establish a connection between the self-modelling problem formulated and the piston problem.

We shall assume that the piston is at the point with mass coordinate $x=0$. Then from (3) it follows that on it the self-modelling coordinate $\xi$ is proportional to the time. In the system of coordinates which we are using the wave front $(\xi=0)$ is at rest and the piston is at a distance from it, moving in the region $\xi>0$ (since $t>0$ ) with constant mass speed $D$ (it is assumed that $D>0$ ) (see Fig. 1). Defining the profile $\bar{\eta}=\bar{\eta}^{\prime}(\xi)$, from equation (6) with initial condition $\bar{\eta}=\bar{\eta}_{0}=1$, given 
for $\xi=0$, we can find from (5) the profiles $\bar{v}=\bar{v}(\xi)$ and $\bar{T}=\bar{T}(\zeta)$ and in the same way determine the law of variation of the piston speed with time and the law of temperature variation.

Assuming the boundary conditions obtained on the piston and solving system (1), for instance, by the method of finite differences with initial conditions (2), we can calculate the profile of the traveling wave leaving the piston.

\section{Investigation of the solutions for the case $\mathbf{1}<\gamma<\mathbf{3}$}

1. The character of the solution of equation (6) near the initial background (the line $\bar{\eta}=1$ on the plane $(\bar{\eta}, \xi)$ )

We shall consider successively the cases $m>1, m=1, m<1, m=0$, and $m<0$.

(1) If $m>1$ (in Fig. 2 the integral curves shown by the continuous lines correspond to this case), the integral curves for $\eta=1$ have a derivative which becomes $-\infty$ for finite values of $\xi$. The expansion in the neighbourhood of the front $\xi=0, \pi_{1}=1$ takes the form

$$
\bar{\eta}=1-\left(\frac{m}{\gamma-1} \xi\right)^{1 / m}+\ldots
$$

where the terms of higher order are omitted. Hence it follows that $\bar{\eta}^{\prime}(0)<0$ for $m>0$ and any $\sigma$. Therefore the function $\bar{\eta}=\bar{\eta}(\xi)$ decreases as $\xi$ increases in proportion to its distance from the wave front. From (5) it follows that the remaining functions are proportional to $\xi^{1 / m}$, i.e. on the wave front the derivatives of all the functions with respect to $\xi$ are infinite if $m>1$. Here the heat flux, temperature, speed and density near the front increase sharply.

(2) Let $m=1$ (see the line shown in Fig. 2 by dashes with one dot). Then the derivative $\bar{\eta}^{\prime}(0)$ is finite and $\bar{\eta}_{\xi=0}=-1 /(\gamma-1)$.

(3) If $0<m<1$ (see dashed line in Fig. 2) the first derivatives on the wave front are zero.

From (7) it follows that if $m=1 / k$ (where $k$ is a positive integer) at the point $\bar{\eta}=1, \xi=0$ the derivatives up to the $k$-th inclusive vanish, 
and beginning with the $(k+1)$-th the derivatives have an infinite discontinuity. Thus the smoothness of contact of the 1 ine $\bar{\eta}=1$ with the integral curves increases as $m \rightarrow 0,(k \rightarrow \infty)$.

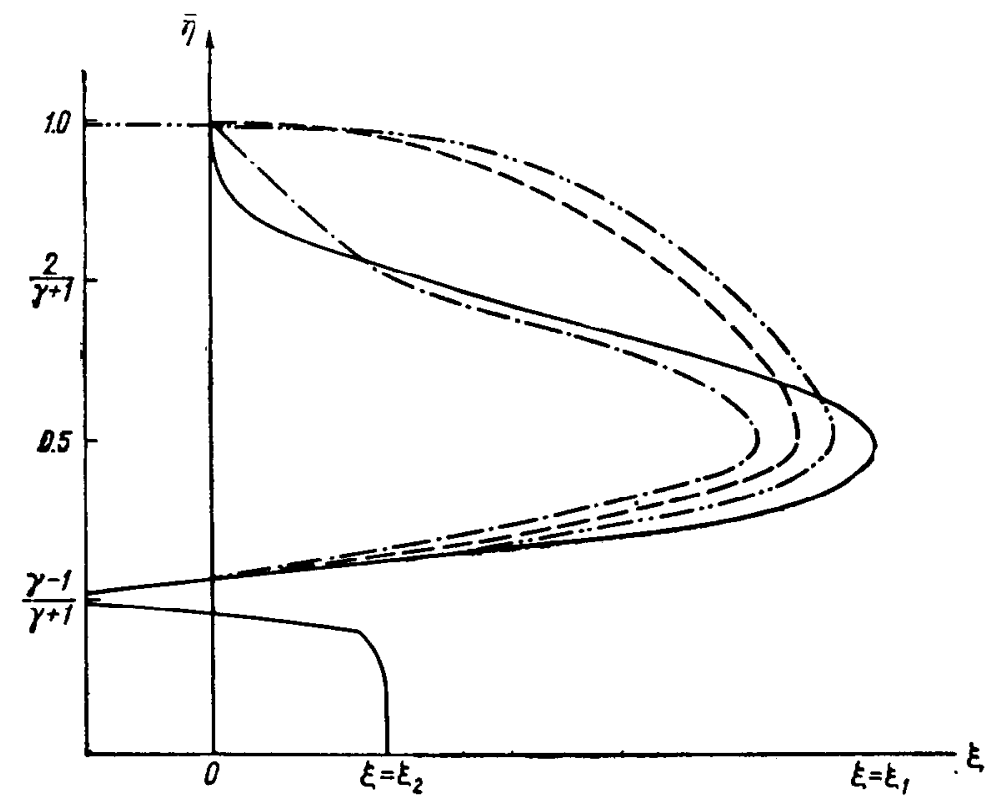

FIG, 2 .

(4) If $m=0$ the expansion near $\bar{\eta}=1$ has the form

$$
\bar{\eta}=1-C e^{[1 /(\gamma-1)] \xi}+O\left[(1-\bar{\eta})^{2}\right],
$$

where $C$ is an arbitrary constant. In this case the integral curves only approach the line $\bar{\eta}=1$ asymptotically as $\xi \rightarrow-\infty$.

(5) For $m<0$ the expansion does not exist if $\xi=0$ but has meaning as $\xi \rightarrow-\infty$. Hence it follows that for $m \leqslant 0$ the integral curves cannot intersect the line $\bar{\eta}=1$ for finite values of $\xi$. In Fig. 2 the dashed line with two dots corresponds to this case.

2. The general character of the field of integral curves

In Section 1 it was shown that in the neighbourhood of the point $(\bar{\eta}=1, \xi=0)$ the function $\bar{\eta}=\bar{\eta}(\xi)$ can only decrease. This means that if $1<y<3$ only compression and heating waves are possible. The monotonic decrease in $\bar{\eta}$ with increase in $\xi$ is confined to the region 
$0.5<\bar{\eta} \leqslant 1$. The line $\bar{\eta}=0.5$ in intersected by the integral curve, leaving the line $\bar{\eta}=1$, at some point $\left(\xi=\xi_{1} ; \vec{\eta}=0.5\right)$ of the plane $(\xi, \bar{\eta})$. The derivative $\left(\bar{\eta}^{\prime}\right)_{\xi_{1}-0}$ becomes $+\infty$ at this point. In the strip $a<\bar{\eta} \leqslant 0.5$, where $a=(\gamma-1) /(\gamma+1)$, we have $\bar{\eta}^{\prime}>0$. This means that after the intersection with the line $\bar{\eta}=0.5$ the integral curves turn towards the side of decreasing values of $\xi$ and also approximate to the line $\bar{\eta}=a$. The line $\bar{\eta}=a$ is a solution of equation (6). We can show that the integral curves running in the strip $a<\bar{\eta}<0.5$ and in the strip $0<\bar{\eta} \leqslant a$ do not intersect the line $\bar{\eta}=a$ but approach it asymptotically.

In fact, on linearizing equation (6) near $\bar{\eta}=a$ we obtain

$$
\bar{\eta}=a+\text { const. } e^{\varphi(a) \xi} \text { for } \bar{\eta}>a, \quad \bar{\eta}=a \text { - const. } e^{\varphi(a) \xi} \text { for } \bar{\eta}<a,
$$

where $\varphi(a)=0.5(1-a)^{1-m} a^{-(\sigma+m+1)}(1-2 a)^{-1}, a=(\Upsilon-1) /(\Upsilon+1)$. Consequently if $\bar{\eta} \rightarrow a+0$ and, also as $\bar{\eta} \rightarrow a-0$ the argument $\xi$ increases without limit $(\xi \rightarrow-\infty)$. In the strip $0<\bar{\eta}<a$ we have $\bar{\eta}^{\prime}<0$ and with increase of $\xi$ the values of $\bar{\eta}$ decrease right up to $\bar{\eta}=0$. The line $\bar{\eta}=0$ is intersected by the integral curve at some point $\xi=\xi_{2}$.

The behaviour of the integral curves near the line $\bar{\eta}=0$ will be studied in detail in Section 4.

Note that the integral curves have physical meaning only when the value of $\xi$ increases on them. This is connected with the fact that on the piston $\xi \approx D^{-2 n+2} t$ and therefore the decrease of $\xi$ along the integral curve is equivalent to the reverse movement of time. Thus only a section of the integral curve corresponds to the profile of a travelIing wave in the strip $0.5 \leqslant \bar{\eta} \leqslant 1$, i. e. $0 \leqslant \xi \leqslant \xi_{1}$. In the remaining part of the plane $(\xi, \bar{\eta})$ the extension of the continuous solution is impossible.

3. The basic properties of travelling waves for $\gamma<3$

From the above analysis we can draw the following conclusions.

For $m \leqslant 0$ the wave front exists for $\xi=-\infty$, and for $m>0$ the wave has a front for finite values $\xi=\xi_{0}=0$. This shows that for $m \leqslant 0$ the speed of propagation of heat is infinite. 
If $m>0$ the wave is propagated with finite mass speed $D$. Just as in the case of one equation of non-linear heat conduction, without considering the gas dynamic influence, the physical values are continuous on the wave front.

Note that similar results are obtained on considering one equation of non-linear heat conduction not taking into account the hydrodyramic motion (work of compression).

In Fig. 3 profiles are given of $\bar{T}(\xi), \bar{v}(\xi), \bar{\rho}(\xi)$ and $\bar{u}(\xi)$ obtained as a solution of (6) in the region $n \leqslant \xi \leqslant \xi_{1}$, for $m=4, \sigma=1$ and $\gamma=5 / 3$.

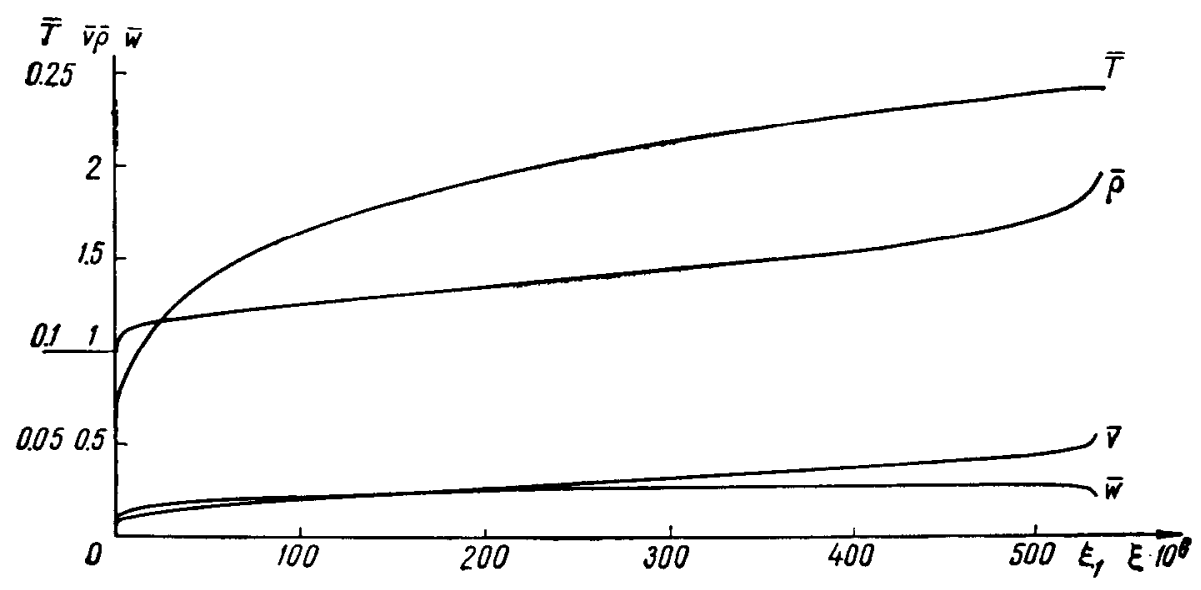

FIG. 3 .

A solution in the form of a continuous travelling wave exists only up to $\xi=\xi_{1}(\bar{\eta}=0.5)$, i.e. only up to a definite instant $t=t$, when a dimensionless critical temperature $\bar{T}_{1}=0.25$ is reached on the piston. Note that in the case of an equation of non-linear heat conduction, without taking into account the hydrodynamic influence, there are no temperature limitations for travelling TW's (see [1]).

The existence of a solution only up to $\xi=\xi_{1}$ can be explained if we consider that the process of heat propagation in the medium generates sound disturbances at the same time. The Lagrangian speed of transformation of state $\left(D_{L}=D / p\right)$ in a travelling wave, however, varies from point to point because $\rho$ changes in the wave. We can show that in the region of a travelling $\mathrm{TW}$ (and on the front itself) $D_{L}$ is larger than the local isothermal speed of the sound disturbances which arise 
$\left(c_{T}=\sqrt{R T)}\right.$. The inequality $D_{L}>c_{T}$ in dimensionless form becomes the condition $\bar{\eta}>\sqrt{\bar{\eta}} \overline{(1-\bar{\eta})}$. As can be easily seen it is satisfied for for $\bar{\eta}>0.5$ which always holds in the region $0 \leqslant \xi \leqslant \xi_{1}$. If $\xi=\xi_{1}$, i.e. if $\bar{\eta}=0.5$, we have $D_{L}=c_{T^{*}}$. Thus in the whole region of the continuous travelling TW which we have constructed $\eta_{L}>c_{T}$, 1.e. such a TW is supersonic (heat is propagated more quicly than sound disturbances). Following [6] we shall call it a temperature wave of the first kind (TW-I). The difference $D_{L}-c_{T}$ decreases with distance from the wave front, while at the point $\xi=\xi_{1}$, or at the corresponding instant $t=t_{1}$, the condition $n_{L}=c_{T}$ is not satisfied on the piston. For $t>t_{1}$ the conditions of a traveling wave can be retained in the case where $D$ varies, or inside the travelling wave an isothermal discontinuity occurs which enables us to find a solution with $D_{L}<c_{T}$.

From the expansion near $\xi=\xi_{1}-0$

$$
\begin{aligned}
& \bar{\eta}=0.5+2^{m+0.5 \sigma-2}\left(\frac{3-\gamma}{\gamma-1}\right)^{0.5}\left(\xi_{1}-\xi\right)^{0.5}+\ldots, \\
& \bar{w}=\frac{0.125(3-\gamma)}{\gamma-1}+2^{m+0.5 \sigma-3}\left(\frac{3-\gamma}{\gamma-1}\right)^{0.5}\left(\xi_{1}-\xi\right)^{0.5}+\ldots, \\
& \bar{T}=0.25-2^{2 m+\sigma-4}\left(\frac{3-\gamma}{\gamma-1}\right)\left(\xi_{1}-\xi\right)+\ldots
\end{aligned}
$$

it follows that (1) the temperature $(\bar{T})$ increases in proportion to its nearness to the point $\xi_{1}$; conditions (5) show that at the point $\xi=$ $\xi_{1}(\bar{\eta}=0.5)$ the temperature reaches a maximum value $\bar{T}_{1}=0.25 ;(2)$ near $\xi=\xi_{1}$ as $\xi$ approaches $\xi_{1}-0$ the value of the flux $\bar{w}$ decreases; on the other hand near the wave front (with $\xi=0$ ) the flux increases with increase in $\xi$; consequentiy the function $\bar{w}=\bar{w}(\xi)$ is not monotonic in the interval $0<\xi<\xi_{1}$; the maximum value of the flux $\bar{w}_{m}=0.5 /\left(\gamma^{2}-1\right)$ is reached at the point $\xi$ for which $\eta=\gamma /(\gamma+1)$; (3) the extension of the solution continuously into the region $\xi>\xi_{1}$ is impossible if $1<\gamma<3$.

\section{Investigation of the selution in the case where $r \geqslant 3$}

1. The field of integral curves with $\gamma=3$

The relative position of the lines chosen by us on the plane $(\xi, \bar{\eta})$ changes according to the value of $\gamma$ (see Fig. 2). If $\gamma=3$ the lines $\bar{\eta}=2 /(\gamma+1)$ and $\bar{\eta}=(\gamma-1) /(\gamma+1)$ merge with the line $\bar{\eta}=0.5$, 
i.e. for $\gamma=3$ the region $2 /(\gamma+1)>\bar{\eta}>(\gamma-1) /(\gamma+1)$ disappears and the line $\bar{\eta}=0.5$ ceases to be a line of bending of the integral curves.

For $\gamma=3$ equation (6) takes the form

$$
\bar{\eta}^{\prime}=-0.5 \bar{\eta}^{-(\sigma+m)}(1-\bar{\eta})^{1-m} \text {. }
$$

Hence it follows that $\bar{\eta}^{\prime}$ does not change sign in the region $1>\bar{\eta}_{\eta}>0$ and the integral curves, beginning with $\bar{\eta}=1$, reach the line $\bar{\eta}=0$. on the line $\bar{\eta}=(\sigma+m) /(\sigma+2 m-1)$ there is an inflexion of the integral curves (Fig. 4).

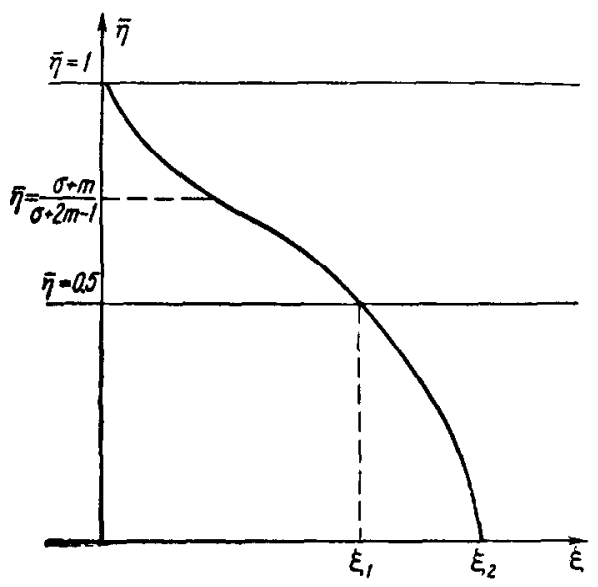

FIG. 4.

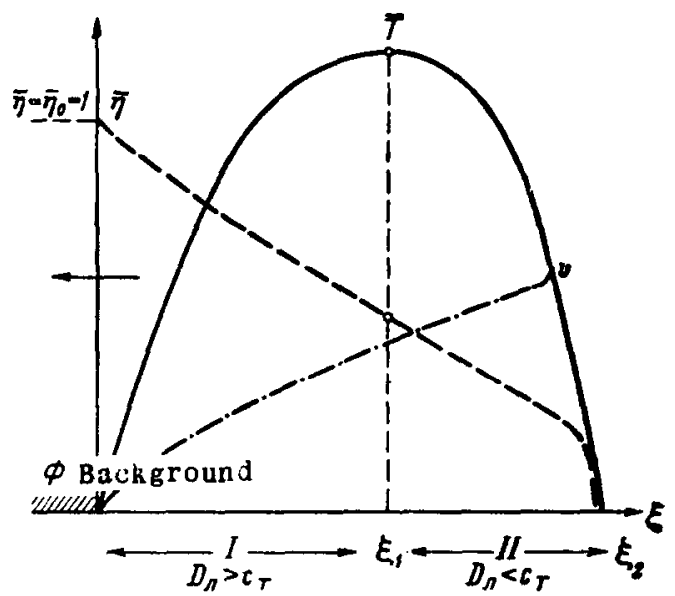

FIG. 5.

The character of the behaviour of the integral curves near the background $(\bar{\eta}=1$ ) remains the same as in the case where $\gamma<3$ (see section 3).

We shall consider the behaviour of the integral curves near the line $\bar{\eta}=0$. If $m+\sigma \neq-1$ the expansion near the point $\left(\xi=\xi_{2}-0, \bar{\eta}=0\right.$ ) to within terms of the second order of smallness has the form

$$
\bar{\eta}=\left[2^{m+2}(\sigma+m) \frac{3 \gamma-1}{\gamma-1}\right]^{1 /(m+\sigma+1)}\left(\xi_{2}-\xi\right)^{1 /(m+\sigma+1)}+\ldots
$$

In the expansion, for generality, the arbitrary quantity $\gamma$ is retained.

From (11) and (12) it follows that: 
(a) for $m+\sigma>-1$ the integral curves intersect the line $\bar{\eta}=0$ at the finite point $\xi=\xi_{2}, \bar{\eta}=0$, while $\left(\overline{\eta^{\prime}}\right)_{\bar{n}=0}=-\infty$ for $m+\sigma>0$, $\left(\overline{\eta^{\prime}}\right)_{\bar{\eta}=0}=-0.5$ for $m+\sigma=0$ and $\left(\bar{\eta}^{\prime}\right)_{\bar{\eta}=0}=0$ for $0>m+\sigma>-1$;

(b) if $m+\sigma \leqslant-1$ the integral curves only approach the line $\bar{\eta}=0$ asymptotically as $\xi \rightarrow+\infty$ while for $m+\sigma=-1$ the expansion near $\vec{\eta}=0$ has the form $\bar{\eta} \simeq C e^{-0.5 \bar{s}}+\ldots$

2. The basic properties of a travelling wave in the case where $\gamma=3$

(1) In Section 3 it was shown that $D_{L}=c_{T}$ at the point $\xi=\xi_{1}(\bar{\eta}=$ $0.5)$. For the case where $y<3$ the solution in the form of a travelling wave existed only up to $\xi \leqslant \xi_{1}$ (up to the instant $t \leqslant t_{1}$ corresponding to the appearance on the piston of the temperature $\bar{T}_{1}=0.25$ ). In the case where $y=3$ the solution, in the form of a continuous travelling wave is also extended into the region $\xi>\xi_{1} \vec{\eta}<0.5$ ) right up to the point $\left(\xi=\xi_{2}, \bar{\eta}=0\right)$.

(2) The character of the profile of the temperature $\bar{T}$ and of the specific volume $\bar{\eta}$, pertaining to the travelling wave with $\gamma=3$, is shown in Fig. 5.

It follows from Fig. 5 that the temperature profile in the travelling wave is non-monotonic.

In the region $1\left(0<\xi<\xi_{1}\right)$ the temperature increases with increase in $\xi$. In this region $\eta>0.5$ and therefore $D_{L}>c_{T}$, i.e. the conditions for a $\mathrm{TW}-\mathrm{I}$ are realized.

In the region $2\left(\xi_{1}<\xi<\xi_{2}\right)$ the temperature falls with increase of $\xi$. The values of $\eta$ vary within the limits $0<\bar{\eta}<0.5$ and therefore $D_{L}<c_{T}$. Following [6] we shall call the conditions of propagation of heat, together with the condition $D_{L}<c_{T}$, the conditions for a temperature wave of the second kind (TW-II). From this point of view we can say that in region 2 a cooling TW-II is realized.

(3) Since the values of $\bar{\eta}<0$ have no physical meaning, the travelling wave considered exists only up to the instant $t=t_{2}$ when the density on the piston becomes infinite $(\bar{\eta}=0)$. The expansion near the value $\xi=$ $\xi_{2}-0$ to within terms of the second order of smallness is of the form

$$
\bar{\eta}=A^{1 /(m+\sigma+1)}\left(\xi_{2}-\xi\right)^{1 /(m+\sigma+1)}+\ldots
$$




$$
\begin{aligned}
& \bar{p}=2-A^{1 /(m+\sigma+1)}\left(\xi_{2}-\xi\right)^{1 /(m+\sigma+1)}+\ldots \\
& \bar{v}=1-A^{1 /(m+\sigma+1)}\left(\xi_{2}-\xi\right)^{1 /(m+\sigma+1)}+\ldots \\
& \dot{\bar{w}}=-0.5+\left(2 A \frac{3 \gamma-1}{\gamma-1}\right)^{1 /(m+\sigma+1)}\left(\xi_{2}-\xi\right)^{1 /(m+\sigma+1)}+\ldots \\
& \bar{T}=(0.5 A)^{1 /(m+\sigma+1)}\left(\xi_{2}-\xi\right)^{1 /(m+\sigma+1)}+\ldots
\end{aligned}
$$

where

$$
A=2^{m+2}(\sigma+m) \frac{3 \gamma-1}{\gamma-1}
$$

From (13) it follows that if $\xi=\xi_{2}$ the dimensionless specific volume and temperature become zero, but the values of the dimensionless speed, pressure and heat flux remain finite.

A travelling wave of the type considered, as can be seen from Fig. 5, is formed on account of the non-monotonic temperature conditions on the piston with monotonic increase in piston speed with time. We must remember that in the system of coordinates (connected with the wave front) which we are considering, the piston moves with constant speed $D$ in the region $\xi>0$.

(4) A cooling TW-II, arizing on the piston at the instant $t=t_{1}$, has its leading front (the point $\xi=\xi_{1}$ ) essentially propagated with finite speed equal to the local isothermal speed of sound. Note that the cooling wave is propagated with finite speed in a mass of gas with temperature differing from zero.

Without a consideration of the influence of hydrodynamics on nonlinear heat conduction, such travelling waves, which are non-monotonic with respect to the temperature, do not exist.

We can give a qualitative explanation of the finite speed of cooling with a temperature background which is different from zero, basing it on the influence of the term corresponding to the work done by the pressure in the energy equation. The work done in compressing the gas by the piston in the case under consideration gives the sources of heat distributed in a cooling wave in such a way that, as the solution shows, they make its speed of propagation finite.

(5) From this point of view a heating TW-2 must be a rarefaction wave, so that on account of the presence of negative work due to compression it has a finite speed for the heat with respect to the non-zero background 
temperature. Note that in the heating TW-2 conditions, constructed in [6], there was in fact a fall of density in the wave proportional to the increase in its temperature. Similar phenomena are observed in the numerical consideration of a number of non-selfmodelling problems.

In this connection there are grounds for assuming that in the conditions of heat propagation of kind $2(\mathrm{TW}-2)$ the strong mutual influence of thermal and hydrodynamic processes makes possible the propagation of

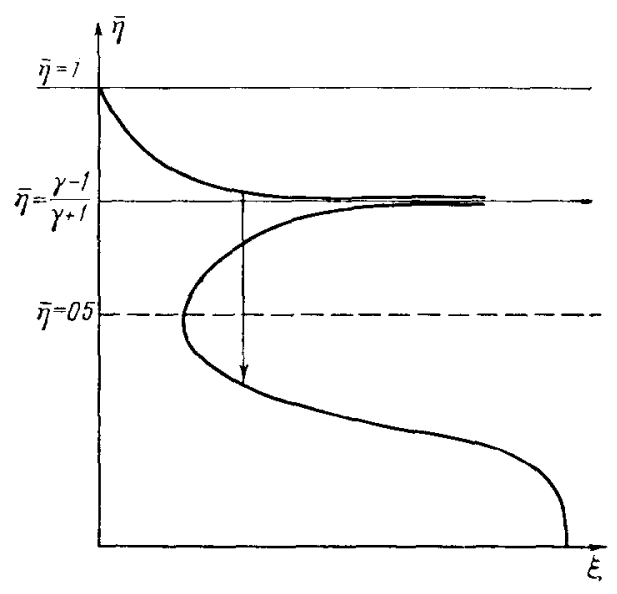

FIG. 6 .

temperature waves with finite speed relative to the non-zero temperature background. We shall return to this assumption again in Section 5 when investigating discontinuous travelling waves.

\section{Conditions for a travelling wave with apparent additional mass}

We shall clarify the question as to whether the solution is extended continuously into the region $\xi>\xi_{2}$. This is possible if the line $\bar{\eta}=0$ is a solution of equation (11). A necessary condition is the satisfaction of the inequality $m+\sigma<0$. The integral curve intersects the line $\bar{\eta}=0$ at a finite point $\xi=\xi_{2}(\bar{\eta}=0)$, as shown in Section 2 of Section 3 , only if $m+\sigma>-1$. Therefore if $0>m+\sigma>-1$ the solution can be extended into the region $\xi>\xi_{2}$ in the form of a continuous travelling wave. In this case behind a heating TW-1 and a cooling TW-2, for $t>t_{2}$ a wave of infinite compression is propagated from the piston through the mass of gas. At each point of this wave, as follows from $\left(5^{\prime}\right)$, $\bar{T}=\bar{\eta}=0, \bar{p}=2, \bar{v}=1$, and $\bar{w}=-0.5$.From the existence of a solution in the form of a travelling wave with finite mass speed it follows that 
the mass, incorporated in the wave, increases and remains finite, in spito of the fact that in the whole region of the wave $\bar{\eta}=0$. The solution of such a form can be treated as the adherence of mass to the piston. Because of the infinite density of the adhering mass the adhering layer remains infinitely thin the whole time. In fact, since for $-1<m_{+}+$ $\sigma<0$ the solution in the form of a travelling wave is impossible in the given region, the mass speed $(D)$ of the wave motion remains finite for $\bar{\eta}=0$. Since $D=\rho\left(D_{\mathrm{E}}-V\right)$ and $\rho=\infty$, it follows that $D_{\mathrm{E}}=V$, i. e. the Eulerian speed of the wave with $\bar{\eta}=0$ is the same as the speed of the piston.

This means that the thickness of the adhering mass layer remains zero.

4. The character of the field of integral curves with $\gamma>3$

The behaviour of the integral curves near the initial background $\bar{\eta}=1$ in no way differs from the case examined in Section 2 . The line $\bar{\eta}=(\gamma-1) /(\gamma+1)$, however, is nearer to the wave front than the line $\bar{\eta}=0.5$. For this reason a continuous travelling wave exists uniting the constant solution in the region $\xi<0$ (the initial background in front of the wave) with the constant solution $\bar{\eta}=(y-1) /(\gamma+1)$, established behind the wave as $\xi \rightarrow+\infty$. The field of integral curves corresponding to the case $\gamma>3$ is shown in Fig. 6 .

\section{Composite travelling waves}

Let us put the question: does a solution exist in the region $\xi>\xi_{1}$ in the furm of a travelling wave with an isothermal sW present at the Doint $\xi=\xi_{1}$ or in front of it? Such a type of solution will correspond to a spasmodic variation of the conditions of the function at the instant $t_{p}, 0<t_{p}<t_{1}$.

Writing Hugoniot' 6 .condition and the condition of isothermality on the front of the SW (see [4]) and using the integrals (5) we obtain

$$
\begin{gathered}
\bar{\eta}_{2}=1-\bar{\eta}_{1}, \quad \bar{v}_{2}=\bar{v}_{0}+1-\bar{\eta}_{2}, \quad \bar{p}_{2}=1-\bar{\eta}_{2}, \\
\bar{T}_{2}=\bar{\eta}_{2}\left(1-\bar{\eta}_{2}\right)=\bar{T}_{1}, \quad \bar{w}_{2}=\frac{1}{2}\left(\frac{r+1}{\Upsilon-1} \bar{\eta}_{2}-1\right)\left(1-\bar{\eta}_{2}\right),
\end{gathered}
$$

where the indices $0,1,2$ are related, respectively, to the value of the front of a TW-1, ahead of the front of a SW, and behind the front of a SW. 
Let us explain the influence will be of the place of the postulation of an isothermal jump behind the front of the TW-1 on the character of the solution which arises behind it.

1. The assumption about the presence of a jump at the point $\xi=\xi_{1}$, where $\bar{\eta}_{1}=0.5$, gives $\bar{\eta}_{2}=0.5=\bar{\eta}_{1}$. Consequently an isothermal jump cannot exist at the point $\xi=\xi_{1}$.

2. If the jump occurs for values $0.5<\bar{\eta}_{1}<2 /(\gamma+1)$, the value of $\bar{\eta}_{2}$ varies within the strip $0.5>\bar{\eta}_{2}>(\gamma-1) /(\gamma+1)$. The function $\bar{\eta}=\bar{\eta}(\xi)$ in this strip increases monotonically with increase in $\xi$ and leads to the point of bending $\xi=\xi_{1}^{\prime}$ (see Fig. 2).

The heat flux, corresponding to the given values of $\bar{\eta}_{2}$, is positive and varies within the limits $1 / 8(3-\gamma) /(\gamma-1)>\bar{w}_{2}>0$.

Thus a composite travelling wave, composed of a TW-1, moving relative to a background with zero temperature, and an isothermal SW, following behind it with positive heat flux behind the front, is unstable. It exists if $0 \leqslant \xi \leqslant \xi_{1}^{\prime}$. The point $\xi=\xi_{1}^{\prime}$ corresponds, on the piston, to the instant $t=t_{1}{ }^{\prime}$, at which $D_{L}=c_{T}$.

3. If $2 /(\gamma+1)<\bar{\eta}_{1}<1$, then from (14) it follows that $0<\bar{\eta}_{2}<$ $(\gamma-1) /(\gamma+),-0.5<\vec{w}_{2}<0$. The integral curves decrease monotonically with increase in $\xi$, reaching the line $\bar{\eta}=0$, and intersect it at the point $\xi=\xi_{2}^{\prime}, \bar{\eta}=0$, where $\left(\eta^{\prime}\right)_{\xi=\xi_{2}^{\prime}}=-\infty$ for $m>1$ (see Fig. 8).

Consequently a composite wave with negative heat flux behind an SW front is also metastable and exists up to the instant $t=t_{2}{ }^{\prime}$, for which $\bar{\eta}=0$, i.e. there is infinite compression with finite pressure on the piston. A detailed analysis of the solution near the line $\bar{\eta}=0$ is given in Section 3 .

4. Suppose, finally, that the jump occurs for $\bar{\eta}_{1}=2 /(\gamma+1)$. As a result of the jump we find ourselves on the line $\bar{\eta}=(\gamma-1) /(\gamma 51)$ (see Fig. 7). All the values behind the SW front satisfy the inequalities

$$
\bar{\eta}_{2}=\frac{\gamma-1}{\gamma+1}, \quad \bar{T}_{2}=\frac{2(\gamma-1)}{(\gamma+1)^{2}}, \quad \bar{v}_{2}-\bar{v}_{0}+\frac{2}{\gamma+1}, \quad \bar{w}_{2}=0
$$

The composite travelling wave in this case brings about a transition from one constant solution to another. We can consider it as a 
generalization of the well known Becker wave (see [8]) for the case of non-linear heat conduction.

The solution obtained enables us to answer accurately the question of the width of the SW front "spread" by the non-linear heat conduction in the case where the background ahead of the $T W$ is zero.

The width of the SW front (in $\mathrm{cm}$ ) has the form

$$
\Delta u=\xi_{\mathrm{ju}} K_{0} D^{2 m-1} R^{-(m+1)} \rho_{0}-2(m-1),
$$

where $\xi_{j u}$ is the self-modelling coordinate of the jump.

In Fig. 7 self-modelling profiles are given behind the jump for three types of composite waves for the case where $m=4, \sigma=1$ and $\gamma=5 / 3$.

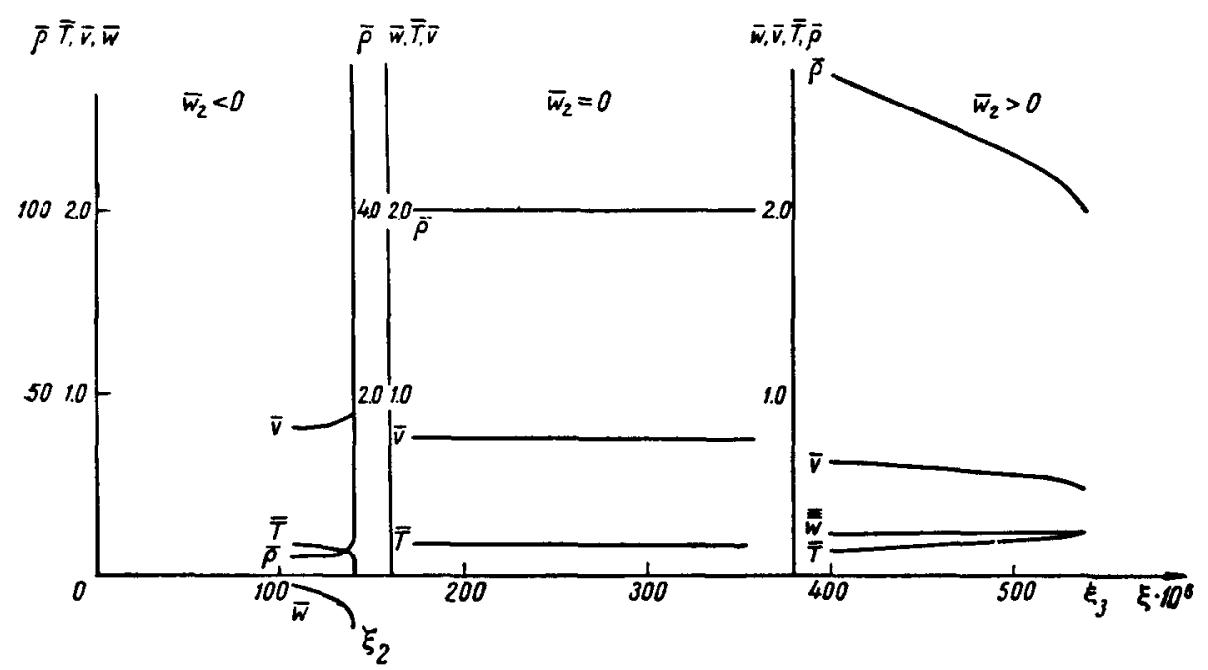

FIG. 7.

In the region behind the isothermal jump we always have $\bar{\eta}_{2}<1$ and so $D_{L}>c_{T}$. Therefore in the case of composite waves with negative heat flux behind the jump $\left(\bar{w}_{2}<0\right)$ in the region behind the jump there is a cooling TW-2.

From Fig. 7 it follows that in a cooling TW-2 the speeds, densities, pressures and heat fluxes (negative) increase with distance from its front.

In the case of a positive heat flux behind the isothermal jump $\left(\bar{w}_{2}>0\right)$ 
conditions of a heating TW-2 occur.

From Fig. 7 it follows that in a heating TW-2 the heat flux increases and the density, speed and pressure decrease with distance from the wave front.

Thus in the case of composite travelling waves we successfully obtain regions in the wave both with conditions of a cooling TW-2 and a heating TW-2. In these cases we can also speak of the finite speed of heat propagation relative to the non-zero background in $\mathrm{TW}-2$ conditions, since the presence of a cooling TW-2 or a heating TW-2 does not adversely affect the structure of a travelling TW-1, going ahead of them.

\section{Some analytic solutions}

1. An analytic solution for integral $m$ and $\sigma($ with $m>1, \sigma>0$ )

For integral $m$ and $\sigma$ and for the conditions $m>1, \sigma>0$ we can obtain an analytic solution* of equation (6)

$$
\begin{aligned}
\xi-\xi_{0}= & \sum_{k=0}^{m+\sigma-1} \sum_{l=0}^{m-1} 2 C_{m+\sigma-1}^{k} C_{m-1}^{l} \frac{1}{2 m+\sigma-2-k-l}(-1)^{m-1+l} a^{k+1} \times \\
& \times(1-a)^{l}(1-2 a)\left[(\bar{\eta}-a)^{2 m+\sigma-2-k-l}-\left(\bar{\eta}_{0}-a\right)^{2 m+\sigma-2-k-l}\right]- \\
& -\sum_{k=0}^{m+c-1} \sum_{l=0}^{m-1} 4 C_{m+\sigma-1}^{k} C_{m-1}^{l} \frac{1}{2 m+\sigma-1-k-l}(-1)^{m-1+l} a^{k+1} \times \\
& \times(1-a)^{l}\left[(\bar{\eta}-a)^{2 m+\sigma-1-k-l}-\left(\bar{\eta}_{0}-a\right)^{2 m+\sigma-1-k-l}\right]+ \\
& +2 a^{m+\sigma}(1-a)^{m-1}\left[(1-2 a) \ln \frac{\bar{\eta}-a}{\bar{\eta}_{0}-a}-\left(\bar{\eta}-\bar{\eta}_{0}\right)\right]
\end{aligned}
$$

where $a=(\gamma-1) /(\gamma+1), \bar{\eta}_{0}$ is the value corresponding to the point $\xi_{0}$. Expression (17) can be written in another form

$\xi-\xi_{0}=\sum_{k=0}^{m+\sigma-1} \sum_{l=0}^{m-1} \sum_{i=0}^{2 m+\sigma-1-k-l} 2 C_{m+\sigma-1}^{k} C_{m-1}^{l} C_{2 m+\sigma-1-k-l}^{i} \frac{(-1)^{m-1+l+i}}{(2 m+\sigma-2-k-l)} \times$

- The first two terms in formulae (17) and (18) are obtained for $k \neq$ $m+\delta-1$ and $l \neq m-1$ simultaneously, and the third term for the condition that $k=m+\delta-1$ and $l=m-1$ simultaneously. 


$$
\begin{aligned}
& \times a^{k+i+1}(1-a)^{l}(1-2 a)\left[\bar{\eta}^{2 m+\sigma-2-k-l-i}-\bar{\eta}_{0}^{2 m+\sigma-2-k-l-i}\right]- \\
& -\sum_{k=0}^{m+\sigma-1} \sum_{l=0}^{m-1} \sum_{i=0}^{2 m+\sigma-k-l-1} 4 C_{m+\sigma-1}^{k} C_{m-1}^{l} C_{2 m+\sigma-k-l}^{i} \frac{(-1)^{m-1+l+i}}{2 m+\sigma-1-k-l} \times \\
& \times a^{k+1+i}(1-a)^{l}\left[\bar{\eta}^{-2 m+\sigma-1-k-l-i}-\bar{\eta}_{0}^{2 m+\sigma-1-k-l-i}\right]+ \\
& +2 a^{m+\sigma}(1-a)^{m-1}\left[(1-2 a) \ln \frac{\bar{\eta}-a}{\bar{\eta}_{0}-a}-2\left(\bar{\eta}-\bar{\eta}_{0}\right)\right] .
\end{aligned}
$$

The general form of solution, given by formula (18), shows that the solution is of the form

$$
\xi-\xi_{0}=P(\bar{\eta})+2 a^{m+0}(1-a)^{m-1}(1-2 a) \ln \frac{\bar{\eta}-a}{\overline{\eta_{0}}-a},
$$

where $P(\bar{\eta})$ is a polynomial in $\bar{\eta}$, beginning with the term $4 a(-1)^{m-1} /$ $(2 m+\sigma-2) \bar{\eta}^{2 m+\sigma-1}, a=(\gamma-1) /(\gamma+1)$.

The presence in the solution of the logarithmic term leads to the division of the field of integral curves into two regions, $\bar{\eta}>a$ and $\bar{\eta}<a$. We can only construct the solution, existing in both regions, by means of an integral curve with jumps.

Expression (19) enables us to establish the influence of the parameter $a=(\gamma-1) /(\gamma+1)$ on the type of solution. Thus, for $a=0(\gamma=1)$, $a=1(\gamma=\infty)$ and $a=0.5(\gamma=3)$ the logarithmic term does not enter into the equation and in this case we can construct a continuous solution from $\bar{\eta}=1$ to $\bar{\eta}=0$. If $a<0.5(\gamma<3)$ the sign in front of the logarithmic term is positive and the integral curves tend to $\xi \rightarrow-\infty$, as $\bar{\eta} \rightarrow a-0$ and if $\bar{\eta} \rightarrow a+0$ we have $\xi \rightarrow+\infty$.

For $a>0.5(\gamma>3)$ the sign in front of the logarithmic term is negative and if $\bar{\eta} \rightarrow a-0$ we have $\xi \rightarrow+\infty$ and if $\bar{\eta} \rightarrow a+0$ $\xi \rightarrow-\infty$. In the case where $a>0.5(r>3)$ it is possíble to construct a continuous solution from $\bar{\eta}=1$ to $\bar{\eta}=a$.

We note further that from expression (9), which is valid for any $m$ and $\sigma$, it follows that the exponents $m$ and $\sigma$ have no essential influence on the results enumerated above. They have a strong adverse effect only on the solution near the lines $\bar{\eta}=1$ (wave front) and $\bar{\eta}=0$. 
To obtain a more complete presentation of the peculiarities, brought into the solution by the non-linearity of the equation of heat conduction, we shall consider the solution of (18) in two particular cases:

Case $A$ - with $m=1, \sigma=0$ - the simplest case of non-linear heat conduction;

Case $B$ - with $m=0, \sigma=-1$ - the case of ordinary linear heat conduction.

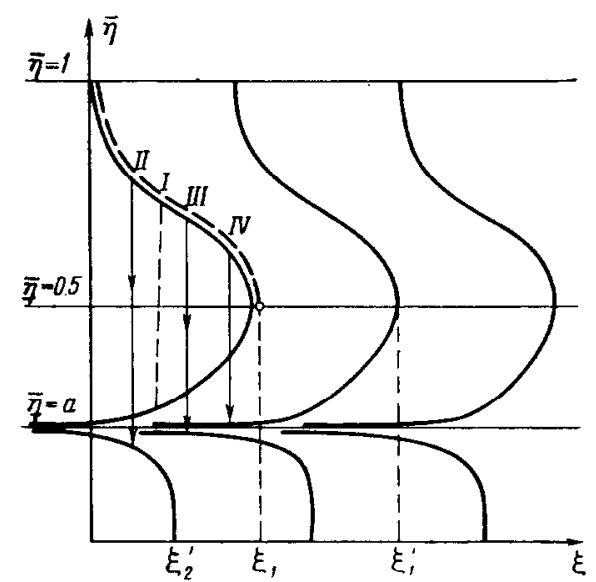

FIG. 8.

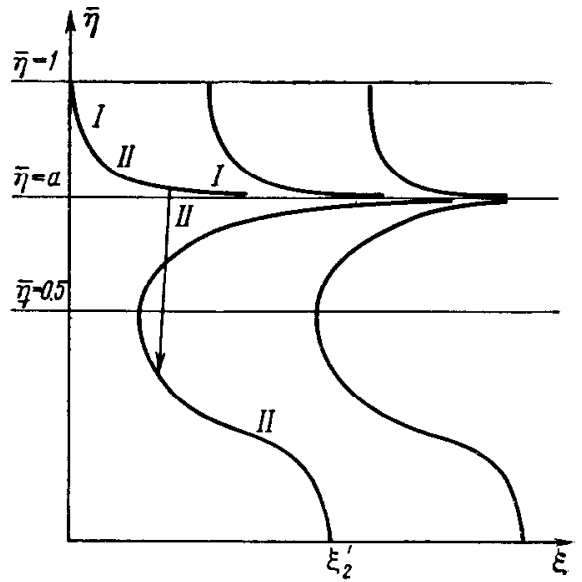

FIG. 9.

2. An analytical solution with $m=1, \sigma=0$ (case A)

From (18) it immediately follows that the solution takes the form

$$
\begin{aligned}
& \xi=2 a(1-2 a) \ln \frac{\bar{\eta}-a}{1-a}+4 a(1-\bar{\eta}) \text { for } \bar{\eta}>a, \\
& \xi-\xi_{2}^{\prime}=2 a(1-2 a) \ln \frac{a-\bar{\eta}}{a}-4 a \bar{\eta} \text { for } \bar{\eta}<a .
\end{aligned}
$$

Hence it is obvious that $\xi_{0}=0$, if $\bar{\eta}_{0}=1$, and $\xi=\xi_{2}^{\prime}$, if $\bar{\eta}=0$.

The field of integral curves for the case $1<y<3(a<0.5)$ is given in Fig. 8. Travelling waves of different types are marked with Roman numerals. Solution 1 is a continuous travelling wave which exists for $0 \leqslant \xi \leqslant \xi_{1}$ (broken line). Solution 2 is a composite travelling wave with $\bar{w}_{2}<0$ behind a front with an isothermal jump.

Note that the jump with $\bar{w}_{2}<0$, depending on the place of its 
formulation, leads to the transition every time to a new integral curve in the region $\bar{\eta}<a$.

Solution 3 occurs with the formulation of the jump for $\overline{\eta_{1}}=2 /(\gamma+1)$ behind the isothermal jump $\bar{w}_{2}=0$, and we pass to the solution $\bar{\eta}=a$.

Solution 4. With the formulation of the jump in the region $\bar{\eta}<2 /(\gamma+1)$ the jump leads us also to a new integral curve in the region $0.5>\bar{\eta}>a$. The solution again exists only up to the point $\xi=\xi_{1}^{\prime}$, where the value $\xi=\xi_{1}^{\prime}$ does not coincide with the similar value of $\xi_{1}$ for solution 1 .

Note that with the formulation of the jump for values very near to $\bar{\eta}=2 /(\gamma+1)$ the values of $\xi_{1}^{\prime}$ and $\xi_{2}^{\prime}$ can be as large as we please, i.e. the conditions of the travelling wave with $\bar{w}_{2}>0$ and $w_{2}<0$ behind the isothermal jump can be extended with a decrease of $\left|\overline{w_{2}}\right|$ to values of $\xi$ as large as we please.

For the case $a>0.5(\gamma>3)$ the field of integral curves is given in Fig. 9.

Solution 1 consists of continuous travelling waves uniting the constant solutions $\bar{\eta}=1$ and $\bar{\eta}=a$.

Solution 2 consists of travelling waves with $\bar{w}_{2}<0$ behind a front with an isothermal jump.

Note that for $a>0.5$ it is impossible to construct a solution with positive flux $\left(\bar{w}_{2}>0\right)$ behind the isothermal jump. In fact, from (14) it is obvious that $w_{2} \leqslant 0$ if $\bar{\eta}_{1} \geqslant 2 /(\gamma+1)$ or, in the case where $\Upsilon \geqslant 3$, for $\bar{\eta}_{1} \geqslant 0.5$, from which it follows that the case $\bar{w}_{2}>0$ is impossible if $\gamma \geqslant 3$.

Finally for the case $a=0.5(\gamma=3)$ the field of integral curves is shown in Fig. 10.

In this case the term witi the logarithm generally drops out, i.e. $\xi=2(1-\bar{\eta})$ or $\bar{\eta}=1-0.5 \xi \quad$ (if $\xi_{l}=0$ ). Hence it follows that $\xi=\xi_{2}^{\prime}=2$ if $\eta=0$. In this case, together with the continuous solution, discontinuous solutions also exist if $\bar{w}_{2}<0$ behind the 
isothermal jump (see Fig. 10 for the solution, with the jump shown by the arrow).

3. The analytical solution for $m=0, \sigma=-1$ (case B)

In the case where $m=0$ and $\sigma=-1$ the solution for $\bar{\eta}>a$ is of the form

$$
\left(\frac{\bar{\eta}-a}{1-a}\right)^{2(1-2 a) /(1-a)} \frac{1}{\overline{\eta^{2}}}(1-\bar{\eta})^{2 a /(1-a)}=e^{\xi},
$$

and for $\bar{\eta}<a$

$$
\left[\frac{(a-\bar{\eta})}{a}\right]^{2(1-2 a) /(1-a)} \frac{1}{\bar{\eta}}(1-\bar{\eta})^{2 a /(1-a)}=e^{\xi} .
$$

The solution is valid for $a>0.5$ and $a<0.5$.

In the case where $a=0.5$ the solution is given by the expression

$$
\frac{1}{\overline{\eta^{2}}}(1-\bar{\eta})^{2}=C e^{\frac{E}{2}} \text {. }
$$

Comparing the solutions for case B with the solution for case A we can see that:

(a) in both cases the picture of the field of integral curves changes equally with change of the parameter $\gamma$;

(b) there are sharp distinctions in the region of the line $\bar{\eta}=1$, where in the case of non-linear heat conduction a finite wave front is observed, and in the case of ordinary linear heat conduction the condition $\eta=1$ is satisfied only if $\xi \rightarrow-\infty$;

(c) similarly near the line $\eta=0$ in case $B$ the integral curves only approach the line $\bar{\eta}=0$ asymptotically (if $\xi \rightarrow+\infty$ ).

Therefore we can say that case $B$ corresponds asymptotically to case $A$. These differences show that the possibility of the existence of a finite width to a shock wave and the appearance in the solution of a characteristic length and characteristic time ( $t_{1}$ or $t_{2}$ etc.) are essentially stipulated by the interaction of hydrodynamics and nonlinear heat conduction and have no place either in the case of purely non-linear heat conduction (without hydrodynamical influence) or in the 
case of hydrodynamics with ordinary linear heat conduction.

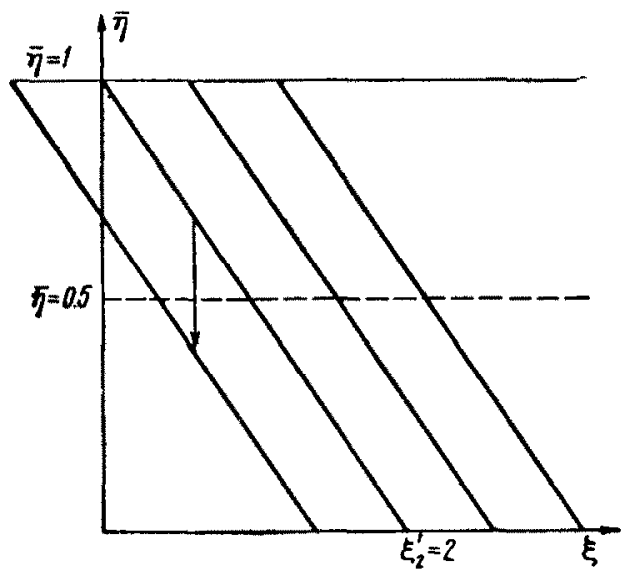

FIG. 10 .

\section{Some numerical solutions}

Using the connection established in Section 1 between the selfmodelling problem of a travelling wave and the similar problem for a piston we give a series of calculations.

1. Calculation of a continuous $T W-1$ existing up to $t=t_{1}$

In Fig. 11 we give the results of a comparison between the selfmodelling solution and the numerical calculation for the system of partial differential equations for a similar problem with a piston.

The continuous Ilnes - the profile of dimensionless temperature (above) and dimensionless density (below) - correspond to the selfmodeling solution. The signs + and $\bigcirc$ show the profile of the corresponding values, found by numerical calculation for a similar problem for a piston at the instant $t=t_{1}$.

The signs - and $X$ show the profiles of the temperature and density obtained on a computer for $t=1.43 t_{1}$ with the condition of invariance for the law of temperature increase, which exists for $t<t_{1}$, and the linear law of increase of speed for the piston in the whole interval $t_{1}<t<1.43 t_{1}$. 


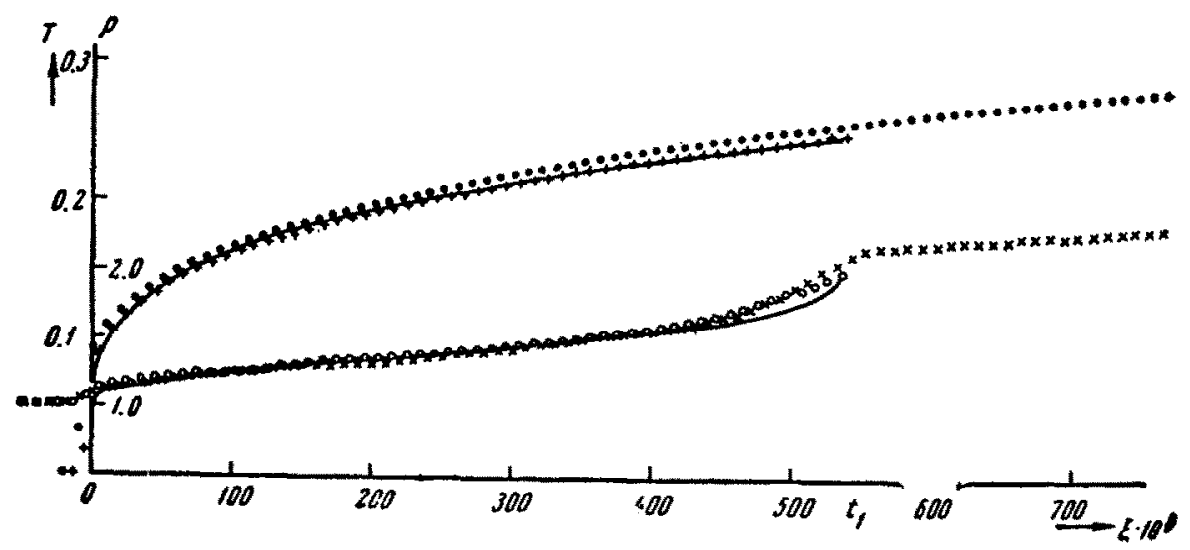

FIG. 11 .

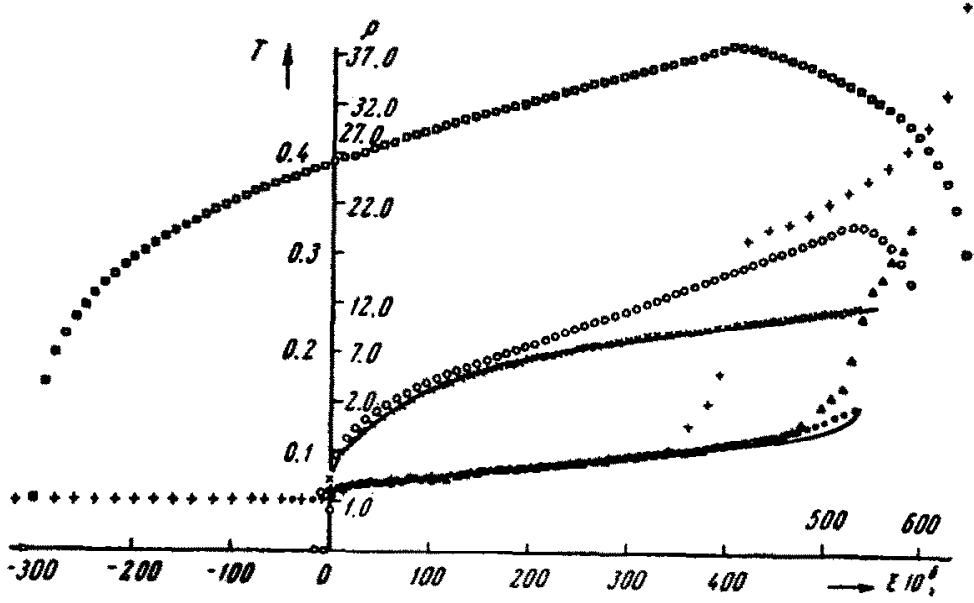

FIG. 12 .

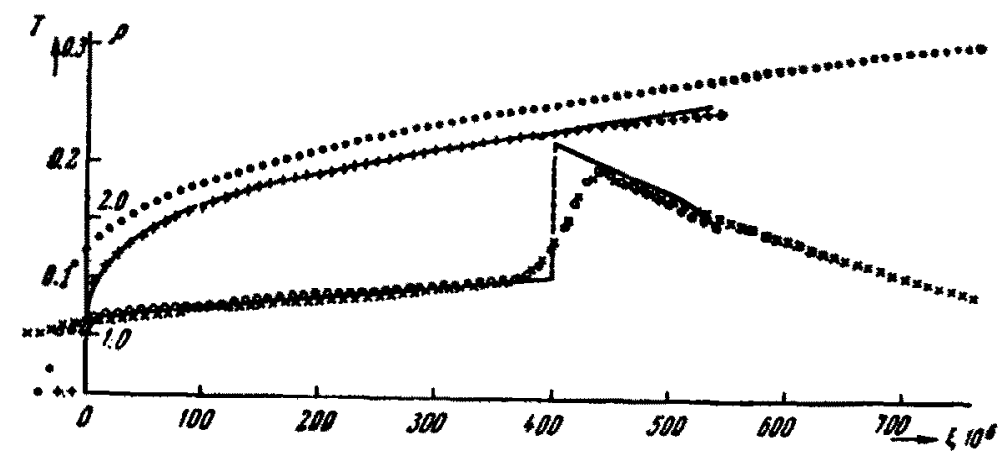

FIG. 13. 
The results obtained show that at the instant $t=t_{1}$ with the conditions for the piston given above there is a variation of speed of propagation of the wave, the characteristic form of the condition for a travelling $\mathrm{TW}-1$ being retained.

2. The calculation describing the appearance inside a travelling TW-1 of an isothermal jump at instants $t>t_{1}$

In Fig. 12 we give results of calculations similar to the case considered in Section 1 Para. 6, but with another method of extending the boundary conditions on the piston at the instant $t>t_{1}$. The temperature increases according to the same law as in Section 1, but the speed of the piston increases four times as quickly. The continuous lines in Fig. 12 show the profiles of the dimensionless temperature (above) and dimensionless density (below), obtained from the self-modelling solution for a travelling wave; the signs $X$ and - indicate the profiles of the respective quantities at the instant $t=t_{1}$, the signs $O$ and $\Delta$ at the instant $t=1.1 t_{1}$, and the signs $\square$ and $t$ at the instant $t=$ $1.20 t_{1}$. With these conditions for $t>t_{1}$ an isothermal $j$ ump occurs in the travelling wave if $\bar{w}_{2}<0$.

3. Lastly, in Fig. 13 we show the calculation for a travelling composite wave with positive flux behind the isothermal jump (TW-1 - TW-2).

The continuous lines - the respective profiles of a dimensionless temperature (above) and density (below) - are obtained from the selfmodelling solution for a composite wave with a positive heat flux behind the jump. The signs + and $O$ indicate the profiles of the respective quantities at the instant $t=t_{1}$ and the signs $\cdot$ and $X$ the same for $t=1.41 t_{1}$. The method of extension consists of retaining the derivative of the temperature and the linear law of increase of speed for $t>t_{1}$.

\section{Discussion of the results}

There is reason to believe that the self-modelling solutions which we have constructed are stable up to the instant $t=t_{1}$. This is confirmed by their coincidence with the numerical solutions of system (1) given in Figs. $11-13$.

An important peculiarity of the discussion is the non-1inearity of the equation of heat conduction which leads to a finite speed of heat propagation relative to the background with zero temperature. This circumstance enables us to consider non-stationary solutions. The 
non-linearity of the equation of heat conduction, taking into account the hydrodynamic influence, leads to the appearance in the solutions of a characteristic length (the distance from the $T W-1$ front to the isothermal jump in composite waves, or for a TW-1 there is a characteristic length corresponding to $t=t_{1}$ ) and the appearance of a characteristic time. Another peculiarity of the constructed solutions is connected with the fact that in a travelling wave the profile is not deformed with time, in spite of the presence of a dissipative process in the medium. Using the invariance of the solution with respect to the transformation of speed it is easy to construct a wave of the type shown in Fig. 14.

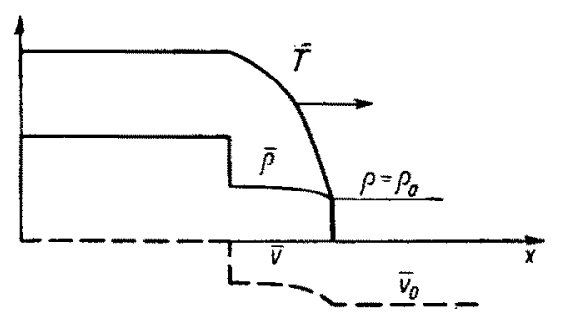

FIG. 14 .

The composite travelling wave with zero flux at the isothermal discontinuity is propagated through a "cold" medium, flowing towards it with constant speed $v_{0}$. This speed decreases partly in the region of the TW and partly at the isothermal jump so that it becomes zero at the jump. Thus in the wave there is a process of transformation of the directed hydrodynamic motion of the medium into thermal motion and, on account of this, the rigid structure (organization) of the wave is maintained.

In solutions of the TW- 1 type the propagation of the state proceeds with supersonic speed. This leads to a weak interaction between the thermal transfer and the hydrodynamic processes. Although the conditions TW-1 exist only up to $t=t_{1}$, but since its time of existence increases in proportion to $D^{2 m-2}$, with a strong dependence of the thermal conductivity on the temperature $(m \gg 1)$ and high speeds of heat propagation $(D \gg 1)$, the condition for $\mathrm{TW}-1$ can be a predominant form of heat outlet.

In solutions of the composite travelling wave type we also observe regions of subsonic heat transfer connected with strong interaction of thermal and hydrodynanic phenomena, conditions of a heating TW-2 and a cooling TW-2. 
A distinct peculiarity of the problems considered is that they are nonadiabatic. Choosing some law of motion for the piston and various laws of change for the heat flux on it we can substantially influence the nature of the connection between the hydrodynamic and thermal processes. The solution with a piston, on which the heat release is given, shows that we can realise the conditions with a strongly squeezed isothermal $S W$ and, in spite of the presence of heat conduction in the medium, can obtain an infinite density peak on the piston.

The authors are grateful to L.N. Dusurina and V.P. Krus for drawing up the programme and performing the calculations on a computer, and also to L. N. Kuk' yanova, A.M. Zakharova and N.G. Teplova who took part in carrying out the calculations and formulating the results.

The authors are indebted to B.L. Rozhdestvenskii for useful advice. Translated by H.F. Cleaves

\section{REFERENCES}

1. ZEL'DOBICH, Ya.B. and KOMPANEETS, A.S. Theory of propagation of heat for heat conduction, dependent on temperature. In "Papers on seventy years of the A.F. Ioffe Academy", Izd-vo Akad. Nauk SSSR, Moscow, $61-71,1950$.

2. BARENBLATT, G.I. Irregular motion of liquid and gas in a porous medtum. Prikl. Mat. Mekh, 16, 6, 67-68, 1952.

3. BARENBlatT, G.I. and VISHIK, I.M. Finite speed of propagation in problems of the non-stationary filtration of a liquid and a gas. Prikl. Mat. Mekh. 20, 3, 411 - 417, 1956.

4. MARSCHAK, $R$. The influence of radiation on the behaviour of shock waves. Physics Fluids, 1, 24-29, 1958.

5. SAMARSKI, A.A, and SOBOL', I.P. Examples of numerical calculations of temperature waves. Zh. vychisl. Mat, mat. Fiz. 3, 4, 702-719, 1963.

6. VOLOSEVICH, P.P., KURDYUMOV, S.P., BUSURINA, L.N. and KRUS, V.P. The solution of a one-dimensional plane problem on the motion of

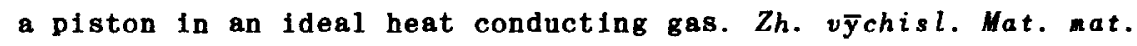
Fiz. 3, 1, $159-169,1963$. 
7. HANIN, M. On Rayleigh's problems on compressible fluids. Q. Jl. Mech. appl. Wath. 13, 2, 184 - 195, 1960.

8. BECKER, B. Stosswell und detonation. Z. Phys, 8, 321 - 362, 1922.

9. ZEL' DOVICH, Ya.B. and RAIZER, Yu.l. The physics of shock waves and high temperature hydrodynamic phenomena (Fizika udarnykh voln i vysokotemperaturnykh gidrodynamicheskikh yablenii). Fizmatgiz, Moscow, 1963. 